\title{
Solar influence on Earth's climate
}

\author{
Rémi Thiéblemont ${ }^{1}$ and Katja Matthes ${ }^{1,2}$ \\ ${ }^{1}$ GEOMAR - Helmholtz Institute for Ocean Research Kiel, \\ Düsternbrooker Weg 20, 24105, Kiel, Germany \\ email: rthieblemont@geomar.de \& kmatthes@geomar.de \\ ${ }^{2}$ Christian-Albrechts Universität zu Kiel, Kiel, Germany
}

\begin{abstract}
Understanding the influence of solar variability on the Earth's climate requires knowledge of solar variability, solar-terrestrial interactions and observations, as well as mechanisms determining the response of the Earth's climate system. A summary of our current understanding from observational and modeling studies is presented with special focus on the "top-down" stratospheric UV and the "bottom-up" air-sea coupling mechanisms linking solar forcing and natural climate variability.
\end{abstract}

Keywords. solar forcing, climate dynamics, stratosphere, ozone, climate modelling

\section{Introduction}

The thermal structure and the composition of the atmosphere strongly depend on solar irradiance. Therefore, fluctuations of the incoming radiation are expected to modulate the Earth's climate and its variability. Although changes in total solar irradiance have been shown to account for a small part of the recently observed global mean temperature changes (IPCC (2013)), there is increasing evidence that variations in solar irradiance are an important source of regional climate variability (Gray et al. (2010), Zhou \& Tung (2010)). For instance, several observational and modeling studies suggested a link between the 11-year solar cycle and surface climate in North America and Europe (Matthes et al. (2006), Ineson et al. (2011)). These results could therefore have significant societal and economical impacts since they would imply an increase of seasonal-to-decadal climate predictability. The attribution and quantification of the climate response to solar variability are however still strongly debated, and require further modeling and observational efforts.

Here we aim at providing a brief overview of the current understanding of the solar irradiance-climate connections on decadal timescales. In section 2 , we review the physical mechanisms that are proposed to describe the influence of total and spectral solar irradiances (TSI and SSI) variations on climate. Section 3 presents the most recent sunclimate modeling outcomes, which have been produced in the framework of the Coupled Model Intercomparison Project Phase 5 (CMIP5). Suggestions for future work are given in section 4.

\section{Mechanisms of solar influences on climate}

The mechanisms proposed to explain the projection of solar irradiance variability onto regional climate are represented in Fig. 1. They are separated into two categories: the "bottom-up" mechanism (box 1), arising from the absorption at the surface of VIS/IR energy variations (yellow arrow), and the "top-down" mechanism (box 2-4), arising from the absorption of UV energy variations in the stratosphere (red arrow). 


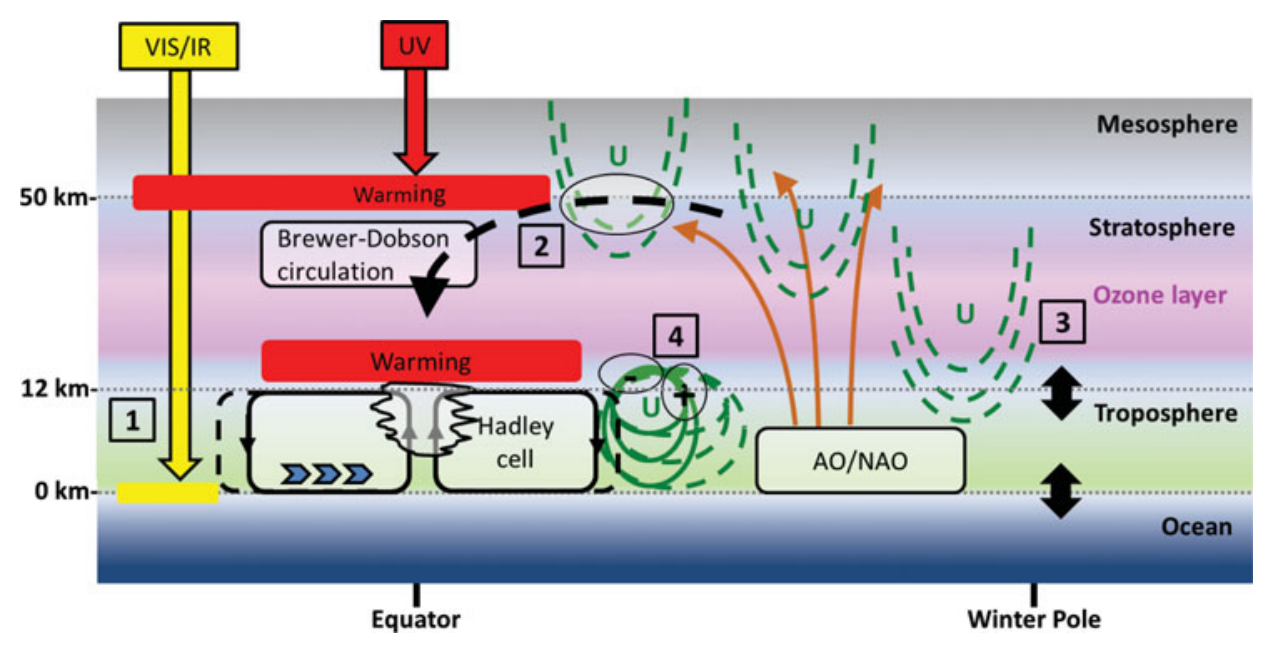

Figure 1. Sketch of the "bottom-up" and "top-down" concepts. See text for details.

Over the course of a solar cycle, the TSI varies by $\sim 1 \mathrm{Wm}^{-2}$, corresponding to a relative variation of less than $0.1 \%$. Despite the small initial perturbation, amplifying dynamical and thermodynamical "bottom-up" mechanisms have been proposed to describe some regional solar effects. It has been suggested that in response to the increased surface heating in subtropical cloud-free regions during solar maxima, the oceans release more water vapor into the atmosphere, which is then transported toward the Inter Tropical Convergence Zone (blue arrows). Water vapor carries latent heat that is released upon condensation, typically in deep convective clouds in the tropics. This release of energy fuels the tropical zonal (Walker) and meridional (Hadley) overturning tropical circulations. The latter is characterized by an ascending branch near the equator and subsidence in the subtropics (black filled stream contours). During solar maxima, a strengthening of the Hadley circulation has thus been proposed (van Loon et al. (2007)). Furthermore, increased subsidence induces even fewer clouds in subtropical regions and more incoming solar radiation, which provide a positive feedback and an amplification of the initial circulation perturbation (Meehl et al. (2008)). The clear solar signals attribution and details of the "bottom-up" mechanism are still under debate, however.

The second possible source of solar-induced climate variability is the fluctuation of UV radiation $(\lambda \sim 200-300 \mathrm{~nm}$ ), which typically varies by $\sim 6 \%$ from solar minima to maxima. Although the relative irradiance variability is much stronger in the UV than in the VIS/IR spectral domain, the UV radiation is absorbed in the upper atmosphere and therefore no direct effect is expected on regional climate. Indirect "top-down" mechanisms are required to explain the transfer of the solar signal from the upper stratosphere to the surface. The starting point of the "top-down" mechanisms is the additional warming in the upper tropical stratosphere under solar maximum phases (red band), due to increased ozone absorption $(\lambda \sim 200-300 \mathrm{~nm})$, and more ozone production through oxygen photolysis $(\lambda<242 \mathrm{~nm})$. The warming anomaly in the stratopause region results in a strengthening of the poleward temperature gradient, which in turn leads to a strengthening of the subtropical westerly jet through thermal wind balance (dashed green contours). These changes in the zonal background flow alter planetary wave propagation (brown filled arrows) and their interaction with the mean flow (transparent ellipse), so that (i) the stratospheric overturning circulation decelerates (black dashed arrow, box 2), and (ii) the initial westerly wind anomalies amplify and propagate poleward and downward through 


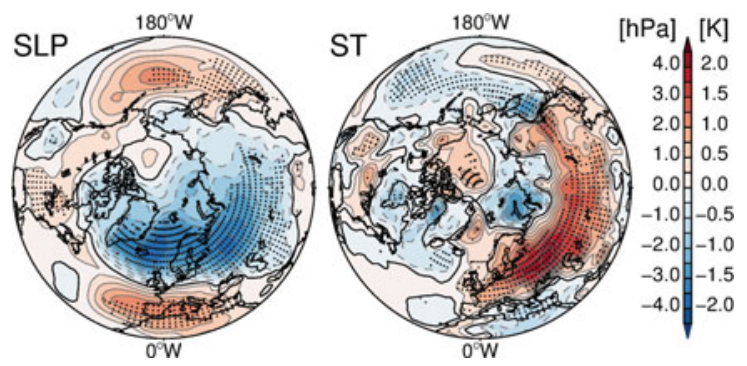

Figure 2. ERA-Interim sea level pressure (SLP) and surface temperature (ST) wintertime anomalies obtained by subtracting solar maximum to minimum phase years between 1958 and 2012. Solar maxima (minima) are defined when the F10.7 index value in winter is greater (lower) than 160 (85) sfu. Stippled areas indicate that the differences are statistically significant.

the winter season (evolution of green contours). The latter phenomenon results in a strengthening of the stratospheric polar vortex (box 3) which ultimately influences the regional climate at mid and high-latitudes through stratosphere-troposphere coupling processes (e.g. Gerber et al. (2012)). This version of the "top-down" mechanism is also known as the "polar route" (Kodera \& Kuroda (2002)).

A "tropical route" has also been suggested, initiated by the secondary warming maximum signal observed in the lower tropical stratosphere (red band). This secondary warming would arise from adiabatic warming combined with increased ozone heating due to the deceleration of the stratospheric overturning circulation. Modeling studies have shown that this temperature anomaly in the lower tropical stratosphere can cause a weakening and a poleward shift of the upper troposphere/lower stratosphere subtropical jet (dashed green contours), as well as an expansion of the Hadley cell (Haigh et al. (2005)).

Both "top-down" approaches have been used to explain the observed surface climate response to solar variability at mid and high latitudes. In particular, they are retained as probable drivers of the Artic Oscillation (AO) and/or North Atlantic Oscillation (NAO) at quasi-decadal timescales, which are the most important modes of variability in the Northern Hemisphere atmospheric circulation in winter (Hurrell et al. (2001)). Fig. 2 shows the observed winter sea level pressure and surface temperature anomalies associated with the 11-year solar cycle. The sea level pressure anomalies are characterized by a decrease of $\sim 3 \mathrm{hPa}$ over the North Atlantic which extends farther over Northern Europe and an increase of $\sim 2 \mathrm{hPa}$ over Euro-Atlantic mid-latitudes region. The associated atmospheric circulation modulation results in warm (cold) anomalies of $\sim 2 \mathrm{~K}(\sim-0.5 \mathrm{~K})$ over Northern Europe (Mediterranean basin). In fact, this pattern strongly resembles the positive phase of the NAO, which induces mild and wet winters in Northern Europe.

The various mechanisms described in this section have been mostly proposed based on observations and/or climate model experiments of reduced complexity in order to minimize the computational costs, e.g., without a complete representation of the stratosphere, or by turning off the interactive ocean-atmosphere coupling. The perception of these mechanisms has however progressively evolved in time. Studies have proposed that the "top-down" may actually act in concert with the "bottom-up" mechanism (Meehl et al. 2009), and that the combination of both ocean-atmosphere couplings and stratospheric processes is crucial to transfer solar irradiance variability into climate variability.

\section{Solar signals in CMIP5 simulations}

Recent CMIP5 historical simulations provided an unprecedented opportunity to assess the ability of the latest generation of climate models to simulate solar-climate 
interactions. Analyses of solar signals in CMIP5 historical simulations have been performed by the Solar Model Inter-comparison Project (SolarMIP) as part of the WCRP/ SPARC-SOLARIS-HEPPA project. SolarMIP addressed (1) the role of ocean-atmosphere coupling (Misios et al. (2015)), (2) the stratospheric dynamical response and its influence on the surface (Mitchell et al. (2015)), and (3) the importance of the ozone solar signal and its feedback on stratospheric dynamics (Hood et al. (2015)). Among all CMIP5 models that conducted historical simulations, 31 models were first selected after excluding those where it was not possible to obtain information on the source of the solar forcing. Among the 31 models, 13 were classified as "high-top", i.e., including a full resolution of the stratosphere, and finally 6 out of the 13 models defined a subgroup which includes interactive ozone chemistry.

The results revealed a globally warmer surface $(\sim 0.07 \mathrm{~K})$ and troposphere in response to solar maxima, that was a robust feature reproduced in the majority of models. The signal maxima showed a lag of $\sim 2$ years consistently with observations. This lag was found to be induced by the extra energy absorbed over solar maxima that is primarily stored in the upper layer ( $\sim 50-100 \mathrm{~m})$ of the ocean (Misios et al. (2015)). Overall, models reproduced a globally average response to solar variability that is similar.

Analysis of solar signals at regional scales depicted less clear results, however. The two regions where solar signals are the most pronounced (i.e. Equatorial Pacific, and the Northern Hemisphere mid and high-latitudes) were examined in detail. Despite a large spread between the different models, evidence of a warmer equatorial central Pacific (with a lag of $\sim 2$ years) and a warmer Arctic were found in response to solar maxima (Misios et al. (2015)). These features showed weak sensitivity to the accurracy of the model stratospheric representation, suggesting the prevalence of the "bottom-up" effect. Conversely, it has been suggested that a good representation of the stratosphere may help to simulate the observed North Atlantic signal, although the magnitude of the signals was weak and the agreement between models relatively low (Mitchell et al. (2015)).

To better understand the discrepancies in the regional response to solar irradiance variability, the stratospheric dynamical response was further investigated. The direct temperature response to increased UV was found to be robust between the 13 different "high-top" models but weaker than in the reanalysis. The resulting "top-down" mechanism was also not consistent accross all models, although some models showed better performance. The latter are those which include a high spectral resolution radiative scheme in the shortwave region (Mitchell et al. (2015)). Interestingly, it was noted that models which include interactive ozone chemistry showed particularly relevant dynamical signals in the stratosphere (Hood et al. (2015)).

In summary, the three studies revealed that the models compare favorably with each other and with the observations/reanalysis at global scale. Conversely, the regional climate and stratospheric responses to the solar cycle were generally found challenging. In the next session, we propose future directions of research which may help to clarify the regional climate and stratospheric aspects.

\section{Outlooks}

Here, we develop three important issues which should be addressed in the near future:

- Model formulation. A rigorous representation of the impact of UV forcing on the atmospheric thermal structure is obtained by using a radiative transfer module which adequately resolves spectral solar variability and by including effects of solar induced ozone variations. The latter can be achieved by (i) prescribing the ozone changes or (ii) interactively calculating the ozone chemistry. While the first option is usually preferred 
as it requires significantly less computational ressources, the second option remains the most accurate way to simulate ozone feedbacks on atmospheric dynamics and radiation which are important for the representation of the solar signal. Note that out of 31 CMIP5 models, only 3 fullfill these characteristics. More modeling sensitivity studies should be conducted to assess the relevance of implementing interactive ozone chemistry.

- SSI forcing. All the CMIP5 models were forced with the NRLSSI (Naval Research Laboratory Solar Spectral Irradiance) conservative solar spectral irradiance model, which represents the lower limit of the magnitude of SSI solar cycle variation among all models and measurements available (Ermolli et al. (2013)). This could partly explain the weaker upper stratosphere solar temperature anomalies compared to reanalyses, and the weak representation of the "top-down" mechanism. For instance, modeling studies using stronger UV forcing than NRLSSI revealed promising stratospheric and regional climate solar signal (Ineson et al. (2011), Scaife et al. (2013)). In this context, it is crucial to assess the best estimate of SSI variability.

- Signals attribution. There is increasing evidence that some other variability sources may alias the solar signals, leading to wrong signals attribution. Chiodo et al. (2014) inferred that the supposed solar response in tropical lower-stratospheric ozone and temperature (Fig. 1) may partly result from aliasing of the solar cycle with the major volcanic eruptions El Chichón and Pinatubo, which by chance occurred following solar maxima in 1982 and 1991. Recently, Thiéblemont et al. (2015) showed that the coupled oceanatmosphere system can itself generate intrinsic quasi-decadal climate oscillations, i.e. without influence of any external forcing, and that solar variability may synchronize this internal variability. These examples highlight that more model sensitivity studies are necessary to precisely identify the role of the various internal or external variability sources in their interaction with the Earth's climate variability.

\section{References}

Chiodo, G., et al. 2014, Atmos. Chem. Phys., 14, 5251-5269

Ermolli, I., et al. 2013, Atmos. Chem. Phys., 13, 3945-3977

Gerber, E. P., et al. 2012, Bull. Am. Meteor. Soc., 93, 845-859

Gray, L. J., et al. 2010, Rev. Geophys., 48, RG4001

Haigh, J. D., Blackburn, M., \& Day, R. 2005, J. Climate, 18, 3672-3685

Hood, L. L., et al. 2015, Q.J.R. Meteor. Soc., doi:10.1002/qj.2553

Hurrell, J. W., Kushnir, Y., \& Visbeck, M. 2001, Science, 291, 603-605

IPCC 2013, Fifth Assessment Report of the Intergovernmental Panel on Climate Change, Cambridge University Press, 1535 pp, doi:10.1017/CBO9781107415324

Ineson, S., et al. 2011, Nat. Geosci., 4, 753-757

Kodera, K. \& Kuroda, Y. 2002, J. Geophys. Res., 107, 4749

Matthes, K., et al. 2006, J. Geophys. Res., 111, D06108

Meehl, G. A., et al. 2008, J. Climate, 21, 2883-2897

Meehl, G. A., et al. 2009, Science, 325, 1114

Misios, S., et al. 2015, Q.J.R. Meteor. Soc., accepted

Mitchell, D. M., et al. 2015, Q.J.R. Meteor. Soc., 141, 2390-2403

Scaife, A. A., et al. 2014, Geophys. Res. Lett., 40, 434-439

Thiéblemont, R., et al. 2015, Nat. Commun., 6:8268

van Loon, H., Meehl, G. A., \& Shea, D. 2007, J. Geophys. Res., 112, D02108

Zhou, J. \& Tung, K. K. 2010, J. Climate, 23, 3234-3248 